\title{
Search for sterile neutrino mixing in the muon neutrino to tau neutrino appearance channel with the OPERA detector
}

\author{
Matteo Tenti ${ }^{*}$ \\ INFN - Sezione di Bologna, I-40127 Bologna, Italy \\ E-mail: matteo.tenti@bo.infn.it
}

The OPERA experiment observed $v_{\mu} \rightarrow v_{\tau}$ oscillations in the atmospheric sector. To this purpose the hybrid OPERA detector was exposed to the CERN Neutrinos to Gran Sasso beam from 2008 to 2012 , at a distance of $730 \mathrm{~km}$ from the neutrino source. Charged-current interactions of $v_{\tau}$ were searched for through the identification of $\tau$ lepton decay topologies. The four observed $v_{\tau}$ interactions are consistent with the expected number of events in the standard three neutrino framework. In this work, we interpret the results in terms of the $3+1$ sterile neutrino model using the GLoBES software. This analysis allows to constrain the effective mixing of the sterile and the new squared mass difference.

XVI International Workshop on Neutrino Telescopes,

2-6 March 2015

Palazzo Franchetti - Istituto Veneto, Venice, Italy

*on behalf of the OPERA Collaboration

† Speaker. 


\section{Introduction}

The OPERA experiment [1] operated on the CERN Neutrinos to Gran Sasso (CNGS) beam [2]. Neutrinos were produced at CERN and directed towards the Gran Sasso Underground Laboratory of INFN (LNGS), $730 \mathrm{~km}$ away, where the detector is located. The experiment is unique in its capability to observe $v_{\tau}$ appearance on an event-by-event basis. Nuclear emulsion films instrumenting the target allow the detection of the short-lived $\tau$ lepton decay, and hence the identification of $v_{\tau}$ Charged Current (CC) interactions [3]. So far, the OPERA experiment observed four $v_{\tau}$ CC interaction candidates, consistent with 2.3 events expected in the standard three neutrino oscillations framework at the so-called atmospheric scale $\left(\Delta \mathrm{m}_{32}^{2} \sim 2.4 \times 10^{-3} \mathrm{eV}^{2}\right.$ [4]) with close-to-maximal mixing. This result represents the first direct observation of $v_{\mu} \rightarrow v_{\tau}$ oscillations in appearance mode [5] and it adds to the consistency of the standard three neutrino oscillations framework.

Anyway, several experimental anomalies exist in neutrino oscillation data that cannot be accommodated in the standard three neutrino oscillations framework (the Gallium $[6,7]$ and nuclear reactor anomalies [8], the LSND [10] and MiniBooNE [9] results) that may hint to the existence of sterile neutrino(s) with a new squared mass difference $\left(\Delta \mathrm{m}_{41}^{2}\right)$ of the order of $1 \mathrm{eV}^{2}$. OPERA can test the sterile neutrino hypothesis and set limits on new effective oscillation parameters looking for deviations from the predictions of the standard three neutrino oscillations model [11, 12].

\section{Analysis in the $3+1$ Neutrino Model}

The number, $\mu$, of expected tau candidate events is evaluated using the GLoBES software $[13,14]$ as:

$$
\mu=N_{b k g}+K \int \Phi_{v_{\mu}}(E) P_{\mu \tau}(E) \sigma_{v_{\tau}}(E) \varepsilon_{v_{\tau}}(E) d E
$$

where $\Phi_{v_{\mu}}$ is the CNGS muon neutrino flux [15], $K$ is a normalization constant which includes the proton on target (pot) and the detector mass averaged on the pot, while $N_{b k g}=0.23$ is the expected number of background events [5]. The cross-section of $v_{\tau}$ CC interaction on ${ }^{208} \mathrm{~Pb}\left(\sigma_{v_{\tau}}\right)$ is evaluated using GENIE [16]. The $v_{\tau}$ identification efficiency $\left(\varepsilon_{v_{\tau}}\right)$ is estimated using the official OPERA simulation and analysis software (OpRelease). The effect of the detector energy resolution is not taken into account since the analysis is based only on the total number of the expected $v_{\tau}$ events and not on their energy distribution.

In presence of a fourth neutrino with mass $\mathrm{m}_{4}$, the $v_{\mu} \rightarrow v_{\tau}$ oscillation probability, $P_{\mu \tau}$, is a function of the $4 \times 4$ mixing matrix $U$ and of three squared mass differences. The parameter $\Delta \mathrm{m}_{21}^{2}$ is fixed to $7.54 \times 10^{-5} \mathrm{eV}^{2}$ and $\Delta \mathrm{m}_{31}^{2}$ is given a Gaussian prior with mean and sigma equal to $(2.47 \pm 0.06) \times 10^{-3} \mathrm{eV}^{2}$ for normal hierarchy of the three standard neutrinos (N.H.) and $(-2.34 \pm 0.06) \times 10^{-3} \mathrm{eV}^{2}$ for inverted hierarchy (I.H.) [4]. Matter effects are also considered, assuming a constant matter density profile (average matter density from the PREM onion shell model of the Earth [19, 20]).

The likelihood $\mathscr{L}$ is defined as: 


$$
\mathscr{L}=P(n \mid \mu) \times \pi\left(\Delta \mathrm{m}_{31}^{2}\right)
$$

where $P$ is a Poissonian distribution depending on $\mu$ and $n$ the number of observed events; $\pi$ is a Gaussian prior of the nuisance parameter $\Delta \mathrm{m}_{31}^{2}$. The effects of possible systematic errors are negligible because, for this analysis, results are dominated by the statistical error.

\section{Analysis Results}

The results of this analysis are given by additional squared mass difference, $\Delta \mathrm{m}_{41}^{2}$, and effective mixing parameter $\sin 2 \theta_{\mu \tau}=2\left|U_{\mu 4}\right|\left|U_{\tau 4}\right|$. Being $\sin 2 \theta_{\mu \tau}$ the leading mixing term at short base line experiments, it allows a direct comparison with previous results of SBL experiment (NOMAD [17], CHORUS [18]). To extract limits on the sterile neutrino mixing parameters, we used the test statistic $-2 \ln \lambda_{p}$, where $\lambda_{p}$ is the profile likelihood ratio [4] defined as:

$$
\lambda_{p}(\alpha)=\frac{\mathscr{L}(\alpha, \widehat{\widehat{\beta}})}{\mathscr{L}(\widehat{\alpha}, \widehat{\beta})}
$$

$\alpha$ represents the parameters of interest $\left(\Delta \mathrm{m}_{41}^{2}, \sin ^{2} 2 \theta_{\mu \tau}\right), \beta$ the nuisance parameters, $\widehat{\beta}$ are the maximum likelihood estimators of $\beta$ and $\widehat{\widehat{\beta}}$ is the value of $\beta$ that maximizes the likelihood for fixed $\alpha$. In order to obtain an exclusion region at $90 \%$ Confidence Level (CL) in the $\left[\Delta \mathrm{m}_{41}^{2}, \sin ^{2} 2 \theta_{\mu \tau}\right]$ plane, the asymptotic $\chi^{2}$ distribution of the test statistic is assumed.

The $90 \% \mathrm{CL}$ excluded region on $\Delta \mathrm{m}_{41}^{2}$ extends down to $10^{-2} \mathrm{eV}^{2}$ for $\sin ^{2} 2 \theta_{\mu \tau}>0.5$. For the normal hierarchy a small region is excluded also at $\Delta \mathrm{m}_{41}^{2} \approx 10^{-3} \mathrm{eV}^{2}$. This stringent limit arises from the suppression of the $v_{\mu} \rightarrow v_{\tau}$ oscillation probability due to the interference with the oscillation probability terms involving sterile neutrino. The analysis was performed assuming $\Delta \mathrm{m}_{41}^{2}>0$. Present limits on the sum of neutrino masses from cosmological surveys do not exclude small negative values for $\Delta \mathrm{m}_{41}^{2}$, the analysis was repeated under this assumption. The exclusion plots obtained in this way are similar to those of Fig. 1, but with hierarchies exchanged.

A separate analysis was performed by deriving an analytical expression for the oscillation probability. Indeed at the OPERA baseline $(730 \mathrm{~km})$ and at the average CNGS neutrino energy $\left(\left\langle E_{v}\right\rangle=17 \mathrm{GeV}\right),\left(\frac{\Delta \mathrm{m}_{21}^{2} L}{4 E}\right)$ is of the order of $10^{-3}$ therefore the dependence of $P_{\mu \tau}$ from $\Delta \mathrm{m}_{21}^{2}$ can be neglected. Moreover the value of $\Delta \mathrm{m}_{41}^{2}$, suggested by the LSND experiment, is of the order of $1 \mathrm{eV}^{2}$, implying that the oscillation terms led by $\Delta \mathrm{m}_{41}^{2}$ can be averaged when taking into account the energy resolution of the OPERA detector (i.e in the one mass dominance approximation with an active small $\Delta \mathrm{m}_{31}^{2}$ [21]). The oscillation probability can then be expressed as: 


$$
\begin{aligned}
P_{\mu \tau} & =\sin ^{2} 2 \psi_{\mu \tau} \sin ^{2}\left(\frac{\Delta \mathrm{m}_{31}^{2} L}{4 E}\right) \\
& +\sin 2 \psi_{\mu \tau} \sin 2 \theta_{\mu \tau} \cos \phi_{\mu \tau} \sin ^{2}\left(\frac{\Delta \mathrm{m}_{31}^{2} L}{4 E}\right) \\
& +\frac{1}{2} \sin 2 \psi_{\mu \tau} \sin 2 \theta_{\mu \tau} \sin \phi_{\mu \tau} \sin \left(\frac{\Delta \mathrm{m}_{31}^{2} L}{2 E}\right) \\
& +\frac{1}{2} \sin ^{2} 2 \theta_{\mu \tau}
\end{aligned}
$$

where $\sin 2 \psi_{\mu \tau}=2\left|U_{\mu 3}\right|\left|U_{\tau 3}\right|$ is an effective angle and $\phi_{\mu \tau}=\arg \left(U_{\mu 3}^{*} U_{\tau 3} U_{\mu 4} U_{\tau 4}^{*}\right)$ is a complex phase, linked to CP violation (i.e. $\sin \phi_{\mu \tau}=J_{\mu \tau}^{C P}$, where $J_{\mu \tau}^{C P}$ is a Jarlskog invariant of the $3+1$ mixing matrix). In order to obtain an exclusion region on the $\left[\phi_{\mu \tau}, \sin ^{2} 2 \theta_{\mu \tau}\right]$ plane, the nuisance parameter $\sin 2 \psi_{\mu \tau}$ has been profiled out, while $\Delta \mathrm{m}_{31}^{2}$ has been fixed to $2.43 \times 10^{-3} \mathrm{eV}^{2}$ for the N.H. and to $2.38 \times 10^{-3} \mathrm{eV}^{2}$ for the I.H., respectively [4]. The result is shown in Fig. 2. By profiling the likelihood also over $\phi_{\mu \tau}$, as shown in Fig. 3, an upper limit of 0.116 is obtained at $90 \% \mathrm{CL}$ on $\sin ^{2} 2 \theta_{\mu \tau}$.

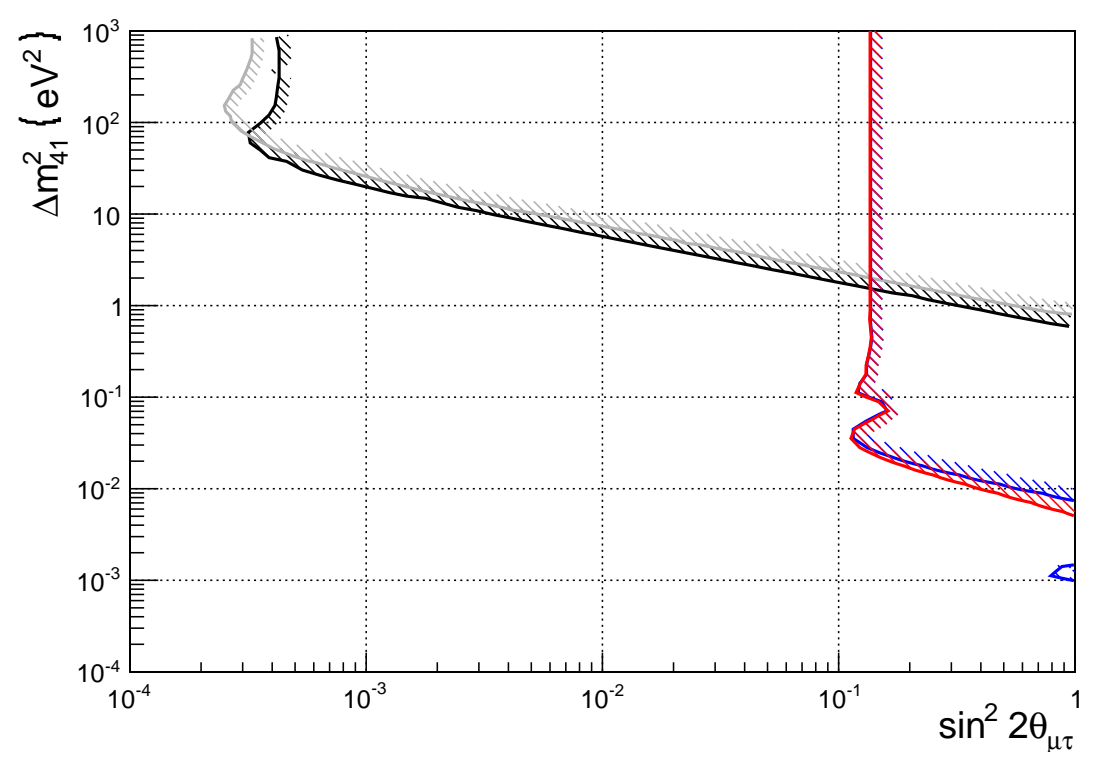

Figure 1: $90 \% \mathrm{CL}$ excluded region in the $\left[\Delta \mathrm{m}_{41}^{2}, \sin ^{2} 2 \theta_{\mu \tau}\right]$ plane for normal (blue) and inverted (red) hierarchy. The $90 \% \mathrm{CL}$ excluded region obtained by CHORUS (black) and NOMAD (gray) experiments are also shown.

\section{Conclusion}

The OPERA experiment was designed to observe $v_{\mu} \rightarrow v_{\tau}$ oscillations through $v_{\tau}$ appearance at a baseline of $730 \mathrm{~km}$ in the CNGS beam. So far, OPERA has observed four $v_{\tau}$ CC candidate interactions, consistent with the expected number of oscillation events in the standard three neutrino 
framework. In this paper we present limits on the existence of a fourth, sterile, neutrino in the $3+1$ neutrino model. At high values of $\Delta \mathrm{m}_{41}^{2}$, the measured $90 \% \mathrm{CL}$ upper limit on the mixing term $\sin 2 \theta_{\mu \tau}$ is 0.116 , independent on the mass hierarchy of the three standard neutrinos. We also extend the exclusion limits on the $\Delta \mathrm{m}_{41}^{2}$ in the $v_{\mu} \rightarrow v_{\tau}$ appearance channel down to values of $10^{-2} \mathrm{eV}^{2}$ at large mixing for $\sin 2 \theta_{\mu \tau} \gtrsim 0.5$.

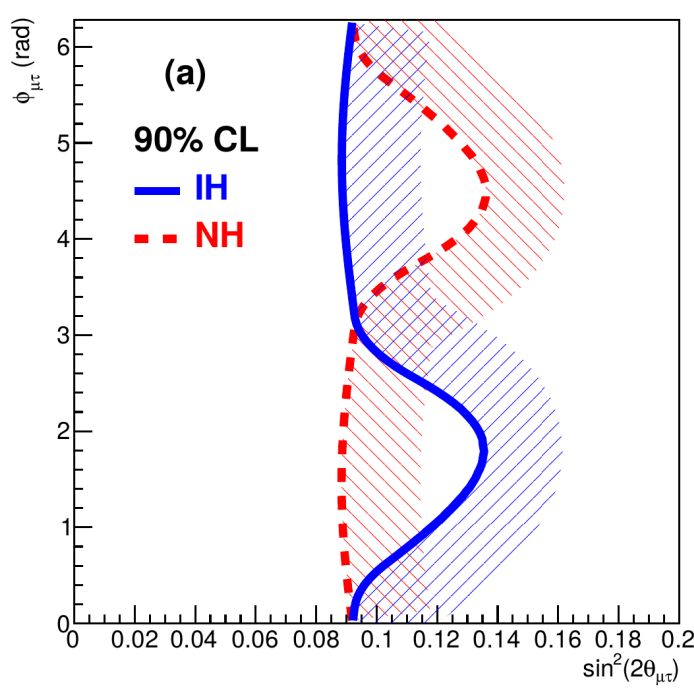

Figure 2: $\quad 90 \% \mathrm{CL}$ exclusion region in $\left[\phi_{\mu \tau}, \sin ^{2} 2 \theta_{\mu \tau}\right]$ plane, for N.H. (dashed red) and I.H. (solid blue) in the one mass dominance approximation with an active small $\Delta \mathrm{m}_{31}^{2}$.

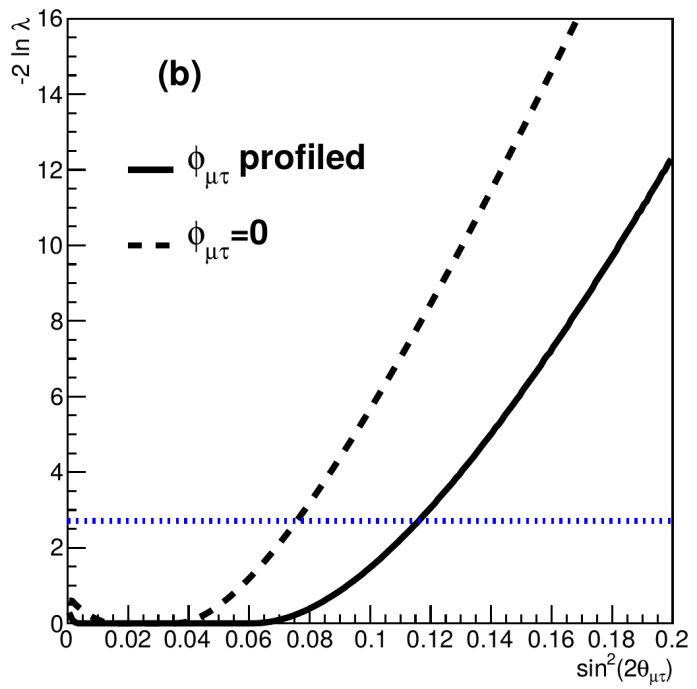

Figure 3: Log-likelihood ratio as a function of $\sin ^{2} 2 \theta_{\mu \tau}$ for $\phi_{\mu \tau}=0$ (dashed line) and for the profile likelihood (continuous line).

\section{References}

[1] R. Acquafredda et al., [OPERA Collaboration], The Opera experiment in the CERN to Gran Sasso neutrino beam, JINST 4 P04018 (2009).

[2] G. Acquistapace et al., The Cern neutrino beam to Gran Sasso (NGS), CERN-98-02, INFN-AE-98-05, CERN-YELLOW-98-02 (1998).

[3] N. Agafonova et al., New results on $v_{\mu} \rightarrow v_{\tau}$ appearance with the OPERA experiment in the CNGS beam, JHEP (2013), 036.

[4] K. A. Olive et al., [Particle Data Group] Review of Particle Physics, Chin. Phys. C86 (2014), 090001.

[5] N. Agafonova et al., [OPERA Collaboration], Observation of tau neutrino appearance in the CNGS beam with the OPERA experiment, Prog. Theor. Exp. Phys. 2014 (2014) 10, 101C01.

[6] M. A. Acero, C. Giunti and M. Laveder, Limits on $v_{e}$ and $\bar{v}_{e}$ disappearance from Gallium and reactor experiments, Phys. Rev. D78 (2008), 073009.

[7] C. Giunti and M. Laveder, Statistical Significance of the Gallium Anomaly, Phys. Rev. C83 (2011), 065504.

[8] G. Mention et al., The Reactor Antineutrino Anomaly, Phys. Rev. D83 (2011), 073006.

[9] A. Aguilar-Arevalo et al., Improved Search for $\bar{v}_{\mu} \rightarrow \bar{v}_{e}$ Oscillations in the MiniBooNE Experiment, Phys. Rev. Lett. 110 (2013), 161801. 
[10] A. Aguilar-Arevalo et al., Evidence for neutrino oscillations from the observation of $\bar{v}_{e}$ appearance in $a \bar{v}_{\mu}$ beam, Phys. Rev. D64 (2001), 112007.

[11] N. Agafonova et al., [OPERA Collaboration], Limits on muon-neutrino to tau-neutrino oscillations induced by a sterile neutrino state obtained by OPERA at the CNGS beam, accepted by JHEP, arXiv:1503.01876v2 [hep-ex].

[12] S. Dusini, G. Sirri and M. Tenti, Search for sterile neutrino mixing in the $v_{\mu} \rightarrow v_{\tau}$ appearance channel with the OPERA detector, http://operaweb.lngs.infn.it/Opera/phpmyedit/notes-pub.php.

[13] P. Huber, M. Lindner and W. Winter, Simulation of long-baseline neutrino oscillation experiments with GLoBES, Comput. Phys. Commun. 167 (2005), 195, hep-ph/ 0407333.

[14] P. Huber, J. Kopp, M. Lindner, M. Rolinec and W. Winter, New features in the simulation of neutrino oscillation experiments with GLoBES 3.0, Comput. Phys. Commun. 197 (2007), 432-438, hep-ph/0701187.

[15] CNGS Neutrino Flux Calculation, http://www.mi.infn.it/ psala/Icarus/cngs.html

[16] C. Andreopoulos et al., The GENIE Neutrino Montecarlo Generator, Nucl. Instrum. Meth. A 614 (2010), 87-104.

[17] P. Astier et al., [NOMAD Collaboration], Final NOMAD results on $v_{\mu} \rightarrow v_{\tau}$ and $v_{e} \rightarrow v_{\tau}$ oscillations including a new search for $v_{\tau}$ appearance using hadronic $\tau$ decays, Nucl. Phys. B611 (2001), 3-39.

[18] E. Eskut et al., [CHORUS Collaboration], Final results on $v_{\mu} \rightarrow v_{\tau}$ oscillation from the CHORUS experiment, Nucl. Phys. B793 (2008), 326-343.

[19] A. M. Dziewonski and D. L. Anderson, Preliminary reference Earth model, Phys. Earth Planet. Interiors 25 (1981), 297-356.

[20] F. D. Stacey, Physics of the Earth, 2nd ed., Wiley, 1977.

[21] Carlo Giunti and Chung W. Kim, Fundamentals of Neutrino Physics and Astrophysics Oxford University Press, 2007. 